JHR

36,6

1104

Received 17 November 2020 Revised 20 January 2021 Accepted 12 February 2021

\section{Women's empowerment and practice of maternal healthcare facilities in Bangladesh: a trend analysis}

\author{
Foyez Ahmmed \\ Statistics, Comilla University, Comilla, Bangladesh
}

\begin{abstract}
Purpose - This study aims to explore whether there is an association between women's empowerment and the utilization of maternal healthcare facilities.

Design/methodology/approach - This study considered four indices for measuring women's empowerment - labor force participation index (LFPI), decision-making power index (DMPI), attitude toward partner's violence index (ATPVI) and knowledge level index (KLI) - and three healthcare facilities number of antenatal visits, delivery with healthcare facilities and postnatal checkup after delivery. Data extracted for this study were from the Bangladesh Demographic and Health Survey 2011 and 2014. A chisquare test was used for bivariate analysis, and a three-level logistic regression model was applied for multivariate analysis.

Findings - An increment was observed in the practice of all considered healthcare facilities, and the percentage of highly empowered women in DMPI decreased from 2011 to 2014 . This study found that higher empowerment of women in DMPI, KLI and ATPVI significantly $(p$-value $<0.05)$ increases the utilization of healthcare facilities. High empowerment of mothers in LFPI was found negatively associated with facility delivery and positively associated with the postnatal checkup.

Originality/value - Women's empowerment was found significantly associated with the utilization of maternal healthcare facilities. This study is seeking the attention of corresponding authority to come up with a more effective intervention program to empower women to utilize maternal healthcare facilities.
\end{abstract}

Keywords Antenatal care, Bangladesh, Facility delivery, Multilevel modeling, Postnatal care, Women's empowerment

Paper type Research paper

\section{Introduction}

The utilization of maternal healthcare facilities plays a pivotal role in promoting maternal healthcare facilities, reducing maternal mortality and child mortality. Maternal mortality refers to the death of a mother due to pregnancy and childbirth-related complications. Although global maternal mortality has dropped noticeably in the past two decades, it remains the leading cause of death among women of reproductive age [1], and international communities are struggling to curb it. The Millennium Development Goal 5 (MDG5) was to reduce maternal mortality, and Bangladesh is one of the few developing countries that have achieved the MDG5 [2] targets. However, Bangladesh is still working toward achieving universal access to reproductive health and utilization of basic maternal healthcare services,

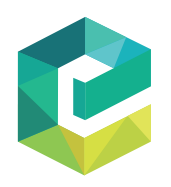

(C) Foyez Ahmmed. Published in Journal of Health Research. Published by Emerald Publishing Limited. This article is published under the Creative Commons Attribution (CC BY 4.0) licence. Anyone may reproduce, distribute, translate and create derivative works of this article (for both commercial and noncommercial purposes), subject to full attribution to the original publication and authors. The full terms of this licence may be seen at http://creativecommons.org/licences/by/4.0/legalcode

The author thanks the Demographic and health survey authority for allowing the use of their data and to the anonymous reviewers for their valuable comments. The author is also grateful to the Comilla University research cell. 
including antenatal care (ANC) by a qualified provider, delivery assistance by a skilled obstetrician and access to a medical facility for childbirth and postnatal care (PNC) from a qualified provider [3].

It has been found that a higher number of ANC visits during pregnancy, facility delivery and postnatal checkup of mothers within two days after delivery reduce maternal mortality to a certain extent [4]. The World Health Organization (WHO) has recommended at least four ANC visits during pregnancy as a part of the global agenda [5]. However, utilization of at least four ANC visits during pregnancy has not yet been realized in Bangladesh. The Bangladesh Demographic and Health Survey (BDHS) 2017 reported that only $47 \%$ of mothers had at least four ANC visits during pregnancy [6].

Also, the prevalence of delivery within a health facility is still halfway toward achieving universal practice. Only $49.6 \%$ of mothers had their deliveries within a health facility according to the report of the BDHS 2017 [6]. The prevalence of PNC of mothers after delivery had similar results with the prevalence of PNC visits for mothers at $52.7 \%$, according to BDHS 2017 [6].

Female empowerment influences the attainment of maternal healthcare facilities $[7,8]$. However, in literature, studies to find the association between female empowerment and utilization of healthcare facilities are scant regarding Bangladesh. Identifying the indices to calculate women's empowerment is a difficult task. There is no strict outline to calculate women's empowerment in literature. We found two studies on Asian and Southeast Asian countries that suggested four components of women's empowerment that we used in our study $[7,8]$. This study aimed to find an association between women's empowerment and the utilization of maternal healthcare facilities. Previous studies identified several socio-economic and demographic factors associated with the utilization of maternal healthcare facilities [9, 10]. Studies showed maternal healthcare facilities varied with different administrative divisions and in the urban and rural areas. Most studies used a single-level logistic regression model for their analysis. But, the results obtained from the logistic regression model could be misleading because of its strict assumptions where data contained a hierarchical structure. Therefore, we used a multilevel modeling approach in our study as BDHS data contain a hierarchical structure.

\section{Methodology}

Data

This study used data sets obtained from the BDHSs 2011 and 2014, two nationally representative cross-sectional studies $[11,12]$. BDHS used a two-stage stratified cluster sampling scheme to collect data. As a sampling frame, BDHS used a list of enumeration areas (EAs) of the preceding population and housing census. In the first stage, EAs are selected as primary sampling units (PSU), with probability proportional to the EA size. In the second stage, a systematic sample of households was selected from each PSU, and each eligible woman of reproductive age between 15 and 49 in the selected households was included in the sample. Further details on sampling and survey procedures were available in publicly available survey reports $[11,12]$. The study sample included 7,095 , and 4,345 currently married women with their last child from 17,842 and 17,863 women included in the BDHS 2011 and 2014, respectively. Therefore, information about 11,440 women concerning their last childbirth was used in this study.

\section{Indices of women's empowerment}

This study used a slightly modified framework of two previous studies for measuring female empowerment $[7,8]$. Four indices - labor force participation index (LFPI), attitude towards 
JHR

36,6 partner's violence index (ATPVI), decision-making power index (DMPI) and knowledge level index (KLI), were considered to measure female empowerment. The LFPI was constructed combining five variables - the respondent had to be working for the past 12 months (no $=0$, yes $=1$ ), who the respondent worked for (not working $=0$, family $=1$, others $=2$, and selfemployed $=3$ ), type of payment received (not working/not paid $=0,1=$ in-kind, $2=$ cash and in-kind, $3=$ cash $)$, type of work $(0=$ not working, $1=$ occasional $/$ seasonal, $2=$ all year $)$, who makes decisions about her earnings $(0=$ not working/not paid, $1=$ husband/others, $2=$ jointly/alone). Categories in a particular variable were ordered in ascending order, i.e. a higher value implied higher empowerment. Then, LFPI was calculated as a sum of the five variables mentioned.

ATPVI was obtained from five questions. Each of the respondents was asked if they would justify wife-beating in the following situations: (1) If she neglects children, (2) if she argues with her husband, (3) if she burns food, (4) if she goes out without her husband's permission, (5) if she refuses to have sex. Responses were "yes (0)" and "no (1)" for each question. Women who responded no in more questions were considered to have a greater sense of entitlement, self-esteem and status, and this reflected positively on her sense of empowerment. ATPVI was calculated as the sum of responses in the above-mentioned five questions.

Four questions were asked to calculate the DMPI: (1) who decides on the respondent's healthcare?, (2) who decides on large household purchases?, (3) who decides on respondent's visiting family or relatives? and (4) who takes the final decision on child healthcare? Each of the four questions had five categories: (1) respondent, (2) respondent and husband/partner jointly, (3) respondent and someone else, (4) husband/partner alone, (5) someone else in the family. For each of the questions, we marked Categories 1, 2 and 3 as 1, which implies women have empowerment in decision-making, and Categories 4 and 5 as 0 , which implies no empowerment. The DMPI was then calculated as the sum of the four binary responses.

The KLI was calculated from two variables: education level and exposure to media. The education level had four categories: no education (0), primary (1), secondary (2) and higher (3). Exposure to media was coded as "yes (1)" if a respondent was exposed to at least one of the three main media forms: newspaper/magazine, television and radio, and "no (0)" if not. Then, the KLI was calculated as the sum of the two variables.

Each of the four indices was converted into a unit-free index between 0 and 1 following the construction method of the human development index as follows:

Dimension Index $=($ Actual value - Minimum value $) /($ Maximum value - Minimum value $)$

After that, each of the four indices was recoded as tertiles with categories labeled as low, middle and high empowered. Confirmatory factor analysis was conducted for the selected 16 variables to see the reliability of the considered four indices. All the four factors showed eigenvalues greater than 1 and factor loadings greater than 0.40. The LFPI accounted for $29.12 \%$ of total variation (Cronbach's alpha $=0.956$ ), ATPVI explained $16.83 \%$ of total variation (Cronbach's alpha $=0.774$ ), decision-making index considered $16.70 \% \%$ of total variance (Cronbach's alpha $=0.833$ ) and finally, women's knowledge level explained $8.55 \%$ of total variation (Cronbach's alpha $=0.631$ ).

\section{Response variables}

This study considered three response variables: number of antenatal visits during pregnancy, facility delivery and postnatal checkup of mothers within two days of delivery. The WHO suggested an agenda of at least four ANC visits for a pregnant mother. So, the number of antenatal visits during pregnancy was categorized as "1" if a mother had at least four ANC visits during pregnancy, and " 0 " otherwise. Place of delivery was considered as a 
proxy variable of facility delivery. Facility delivery was considered as a binary response $(Y e s=1$, No $=0)$. "No" for the respondents who had a delivery at home, and "Yes" for the respondents who had a delivery in public/private hospitals or clinics or non-governmental organization (NGO) clinics. Postnatal checkup of mothers after delivery was considered as a dichotomous variable - "Yes (1)" for the mothers who had a postnatal checkup within 41 days after delivery, and "No (0)" for the rest.

\section{Explanatory variables}

Women's empowerment and healthcare facilities

As explanatory variables besides four indices of women's empowerment, some socioeconomic and demographic factors of mothers and children, namely, place of residence (urban, rural), wealth index (poorest, poorer, middle, rich, richest), sex of child (male, female), parity (1st, 2nd, 3rd, 3+), division (Dhaka, Barisal, Khulna, Chittagong, Rajshahi, Rangpur, Sylhet), wanted index child (yes, no), husband/partner's education level (no education, primary, secondary, higher) and mothers' age $(\leq 20,21-30$, > 30$)$, were considered to see the adjusted effect on response variables. Survey years ( 1 for 2011 , and 2 for 2014) were considered as a quantitative explanatory variable.

\section{Statistical analysis}

For trend analysis, the prevalence of at least four ANC visits, facility delivery and PNC visits were calculated. For bivariate analysis, a chi-square test was used to find the significant association between covariates, and ANC visit, facility delivery and postnatal checkup of respondents. Covariates that were found significant in bivariate analysis were considered in multivariate analysis. Since BDHS data are collected by using a hierarchical structure, it was essential to consider this data collection scheme in our analysis. Avoiding this hierarchical structure in the analysis could result in a misleading conclusion [13]. To consider this hierarchical structure in analysis, the mixed effect modeling approach was used. To see the adjusted effect of covariates on the ANC visit, facility delivery and postnatal checkup of respondents, a three-level logistic random intercept model was considered. The place of residence was considered as a second-level source of variation and division was considered as the third-level source of variation. For example, a three-level logistic random intercept model can be expressed as:

$$
\begin{gathered}
\text { Level } 1: \log \left(p_{i j k} / 1-p_{i j k}\right)=\beta_{0 j k}+\gamma_{1} x_{1 i j k} \\
\text { Level } 2: \beta_{0 j k}=\beta_{00 k}+\alpha_{0 j 0} \\
\text { Level 3 }: \beta_{00 k}=\alpha_{000}+\alpha_{00 k}
\end{gathered}
$$

where $p_{i j k}=\operatorname{Pr}\left(Y_{i j k}=1\right)$ with response variable $Y_{i j k}$. Intra-class correlations (ICC) for the above model has the following formula:

$$
\begin{aligned}
\mathrm{ICC}_{k} & =\frac{\sigma_{k}^{2}}{\sigma_{j}^{2}+\sigma_{k}^{2}+\left(\pi^{2} / 3\right)} \\
\mathrm{ICC}_{J \mid k} & =\frac{\sigma_{j}^{2}+\sigma_{k}^{2}}{\sigma_{j}^{2}+\sigma_{k}^{2}+\left(\pi^{2} / 3\right)}
\end{aligned}
$$

where $\sigma_{j}^{2}$ and $\sigma_{k}^{2}$ are the variances of random effects $\alpha_{0 j 0}$ and $\alpha_{00 k}$ at Levels 2 and 3, respectively. ICC always varies 0 to 1 inclusive. An ICC value greater than 0 implied the presence of hierarchical structure in the data; therefore, it should be considered in the analysis 
JHR 36,6
$[13,14]$. All the analyses were conducted using statistical software IBMSPSS (version 20) and STATA version 15.

\section{Ethical approval}

This study relied on the secondary data sets of the BDHS 2011 and 2014. BDHS was ethically approved by the National Institute of Population Research and Training of the Ministry of Health and Family Welfare, Bangladesh. The survey was conducted maintaining all international ethical standards. All other survey information is publicly available from the website: www.dhsprogram.com. For our study, an online proposal was submitted to the Demographic and Health Survey (DHS) Program, ICF International and a letter of data authorization was obtained. However, an ethical approval code was not provided on this occasion.

\section{Results}

A total of 11,440 mothers from the BDHS 2011 and 2014 were considered in this study. Table 1 shows the percentage distribution of the response variables and covariates for two surveys separately as well as for pooled data. Prevalence of at least four ANC visits, facility delivery and PNC was found to increase from 2011 (26.6, 29 and $45.8 \%$ ) to $2014(32.2,40.2$ and $66.1 \%$ (Figure 1). Percentages of highly empowered women increased for all indices of women empowerment, except the DMPI (Figure 2). In the DMPI, the percentage of highly empowered mothers decreased in $2014(53.5 \%)$ compared to 2011 (56.7\%). Most of the respondents were aged 21 to 30 years, and the ratio of male and female was close to one for both surveys. The percentages of respondents for different wealth index groups were approximately the same for both surveys. The number of rural respondents was greater compared to the number of urban respondents in each survey. An increase was observed in husband's/partner's education levels from 2011 to 2014 . The number of mothers with three or more parity had decreased between the survey years. Most mothers reported that they wanted their child.

The results of the bivariate analysis are reported in Table 2. Chi-square test of association was applied on pooled data to find the factors associated with ANC visits, facility delivery and PNC visit. It was found that the DMPI, KLI and ATPVI were significantly ( $p$-value $<0.01$ ) associated with utilization of ANC visits, and highly empowered women were found to have a higher prevalence of having at least four ANC visits. Other covariates, except for the sex of children, were also found significantly associated with ANC visits ( $p$-value $<0.01)$. All the four indices of women's empowerment were significantly associated ( $\phi$-value $<0.05)$ with facility delivery, and a higher prevalence of facility delivery was found for highly empowered mothers, except in the case of LFPI. All other considered covariates were also significantly associated ( $\phi$-value $<0.01)$ with the utilization of facility delivery. All the four indices and other considered covariates, except the sex of the child, were found significantly associated $(\phi$-value $<0.01)$ with PNC visit within two days of delivery. The prevalence of PNC visits was found higher for highly empowered mothers. Surprisingly, this study found mothers of higher age groups had significantly lower prevalence in all three considered healthcare facilities. Mothers living in urban areas, in wealthier families, mothers whose husbands had higher education and mothers with less parity were found to have a higher percentage of utilization of all considered three healthcare facilities.

Covariates that were significant in the bivariate analysis were considered in the multivariate analysis stage. To consider the hierarchical structure of data, a three-level logistic regression was applied for multivariate analysis using the place of residence and division as Level 2 and Level 3 sources of variation. The results obtained from regression analysis are reported in Table 3. Random effects parameters and ICC values reported in 


\begin{tabular}{|c|c|c|c|c|c|}
\hline Variables & & $\begin{array}{l}\text { Survey 2011 } \\
(N=7,095)\end{array}$ & $\begin{array}{l}\text { Survey 2014 } \\
(N=4,345)\end{array}$ & $\begin{array}{c}\text { Pooled } \\
(N=11,440)\end{array}$ & $\begin{array}{l}\text { Women's } \\
\text { empowerment }\end{array}$ \\
\hline \multirow[t]{2}{*}{ ANC } & $<4$ & 73.4 & 67.8 & 71.3 & \\
\hline & At least 4 & 26.6 & 32.2 & 28.7 & tacilitie \\
\hline \multirow[t]{2}{*}{ Facility delivery } & No & 71.0 & 59.8 & 66.8 & \\
\hline & Yes & 29.0 & 40.2 & 33.2 & \\
\hline \multirow[t]{2}{*}{$\mathrm{PNC}$} & No & 54.2 & 33.9 & 46.5 & 1109 \\
\hline & Yes & 45.8 & 66.1 & 53.5 & \\
\hline \multirow[t]{3}{*}{ Age (years) } & $\leq 20$ & 20.9 & 27.9 & 23.6 & \\
\hline & $21-30$ & 58.8 & 56.6 & 58 & \\
\hline & $>30$ & 20.3 & 15.5 & 18.5 & \\
\hline \multirow[t]{5}{*}{ Division } & Dhaka & 16.8 & 17.7 & 17.2 & \\
\hline & Khulna & 12.0 & 11.7 & 11.9 & \\
\hline & Rajshahi & 13.0 & 12.2 & 12.7 & \\
\hline & Rangpur & 13.2 & 12.4 & 12.9 & \\
\hline & Sylhet & 14.4 & 14.9 & 14.6 & \\
\hline \multirow[t]{2}{*}{ Place of residence } & Urban & 31.6 & 32.2 & 31.9 & \\
\hline & Rural & 68.4 & 67.8 & 68.1 & \\
\hline \multirow[t]{5}{*}{ Wealth index } & Poorest & 20.7 & 20.6 & 20.7 & \\
\hline & Poorer & 19.2 & 18.9 & 19.1 & \\
\hline & Middle & 19.2 & 19.1 & 19.2 & \\
\hline & Richer & 20.0 & 21.2 & 20.5 & \\
\hline & Richest & 20.8 & 20.1 & 20.5 & \\
\hline \multirow[t]{2}{*}{ Sex of child } & Male & 51.8 & 51.6 & 51.7 & \\
\hline & Female & 48.2 & 48.4 & 48.3 & \\
\hline \multirow{2}{*}{ Wanted index child } & No & 29.1 & 25.1 & 27.6 & \\
\hline & Yes & 70.9 & 74.9 & 72.4 & \\
\hline \multirow[t]{4}{*}{$\begin{array}{l}\text { Husband/partner's } \\
\text { education level }\end{array}$} & $\begin{array}{l}\text { No } \\
\text { education }\end{array}$ & 26.6 & 22.6 & 25.1 & \\
\hline & Primary & 29.1 & 30.1 & 29.5 & \\
\hline & Secondary & 30.1 & 31.8 & 30.7 & \\
\hline & Higher & 14.3 & 15.5 & 14.7 & \\
\hline \multirow{4}{*}{ Parity } & $1 \mathrm{st}$ & 33.7 & 40.7 & 36.3 & \\
\hline & 2nd & 30.0 & 29.9 & 30.0 & \\
\hline & 3rd & 17.7 & 15.6 & 16.9 & \\
\hline & $3+$ & 18.6 & 13.8 & 16.8 & \\
\hline \multirow[t]{3}{*}{ LFPI } & Low & 89.1 & 77.4 & 84.6 & \\
\hline & Medium & 0.6 & 2.6 & 1.4 & \\
\hline & High & 10.3 & 20.0 & 14.0 & \\
\hline \multirow[t]{3}{*}{ KLI } & Low & 31.1 & 27.3 & 29.6 & \\
\hline & Medium & 26.5 & 26.2 & 26.4 & \\
\hline & High & 42.5 & 46.4 & 44.0 & \\
\hline \multirow[t]{3}{*}{ ATPVI } & Low & 4.9 & 4.2 & 4.7 & $\begin{array}{l}\text { 1able } 1 . \\
\text { Percentage }\end{array}$ \\
\hline & Medium & 14.9 & 12.5 & 14.0 & distribution of \\
\hline & High & 80.1 & 83.4 & 81.4 & response variables and \\
\hline \multirow[t]{3}{*}{ DMPI } & Low & 30.6 & 33.2 & 31.6 & covariates by survey \\
\hline & Medium & 12.7 & 13.3 & 12.9 & year and for \\
\hline & High & 56.7 & 53.5 & 55.5 & pooled data \\
\hline
\end{tabular}

Table 3 suggested a three-level model was appropriate for analyzing these data as all the random effect parameters were significantly greater than zero and all ICC values were greater than zero. The regression results showed the practice of all three considered healthcare facilities significantly increased in 2014 compared to 2011. Mothers with high and medium KLI were 2.14 and 1.56 times more likely to have at least four ANC visits compared to mothers with low KLI. Mothers with high ATPVI and DMPI also had a higher chance of having at 
Figure 1.

Prevalences of healthcare facilities by survey years
Figure 2. Indices of women's empowerment by survey years
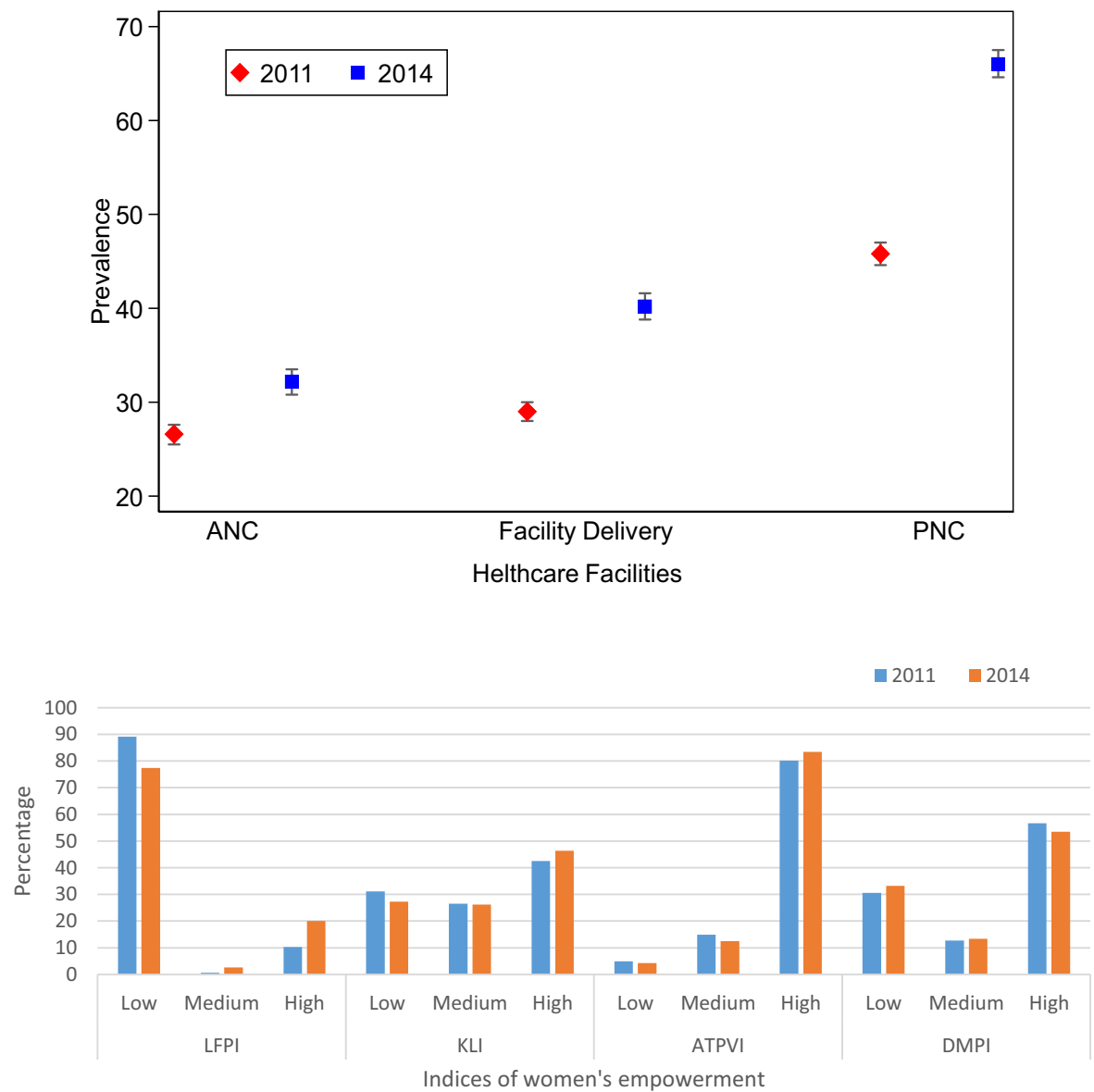

least four ANC visits. Mothers with high values of KLI, ATPVI and DMPI were found more likely to have facility delivery compared to their opposite counterparts. Surprisingly, this study found mothers who were more empowered in the LFPI had a lower chance of facility delivery. However, in the case of a PNC visit, mothers with high empowerment in all four indices were found to have a higher probability of having a PNC visit after delivery. Except for the sex of the child, in the case of the PNC visit, all other covariates that were considered in the regression models were found significantly associated with all three considered healthcare facilities, and the results were similar to the results obtained from the bivariate analysis.

\section{Discussion}

This study found the prevalence of having at least four ANC visits, delivery in healthcare facilities and PNC visits after delivery increased from 2011 to 2014. The increase in the prevalence of PNC visits was higher compared to ANC visits and facility delivery. This increase might be because of the higher prevalence of deliveries at home. The rise in home 


\section{Women's empowerment and healthcare facilities}

1111
Table 2. Prevalence of at least four ANC visits, delivery in a healthcare facility and PNC by survey year and their associated factors with $p$-values obtained from chi-square test using pooled data 
JHR
36,6

1112

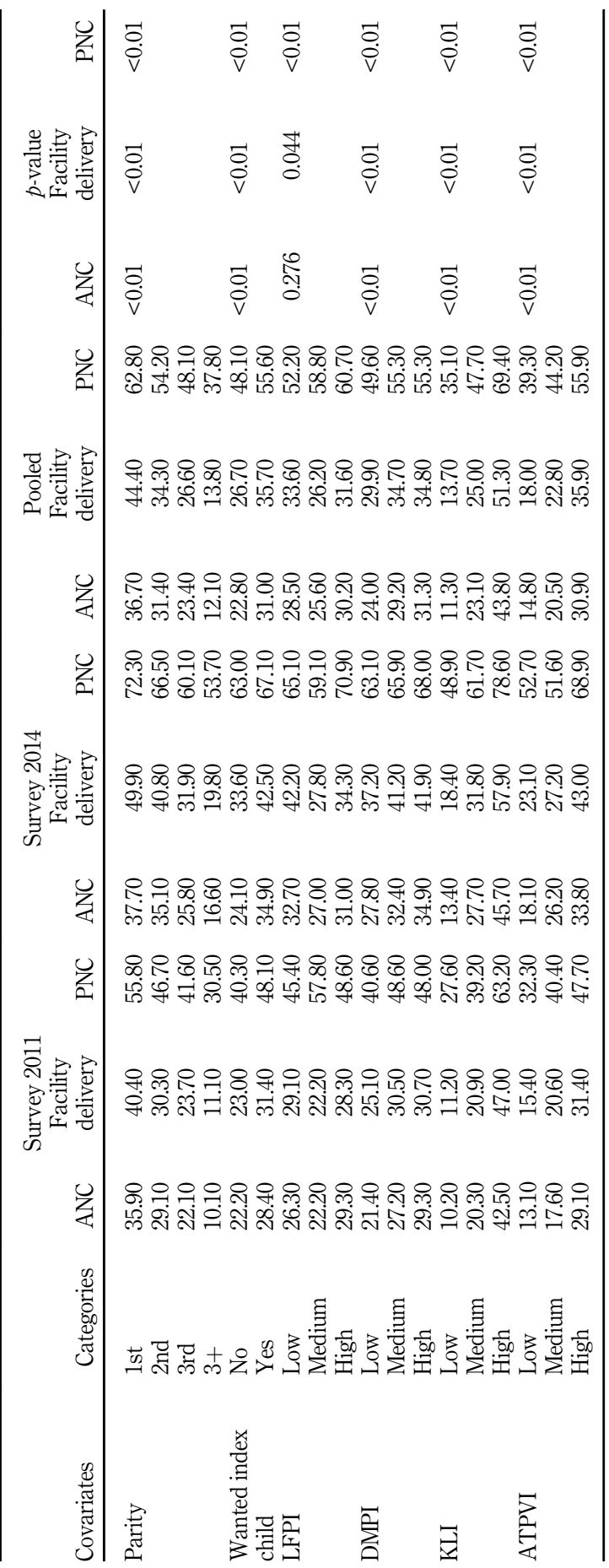




\begin{tabular}{|c|c|c|c|c|c|c|c|}
\hline \multirow[b]{2}{*}{$\underline{\text { Covariates }}$} & \multicolumn{2}{|l|}{ ANC } & \multicolumn{2}{|c|}{ Facility delivery } & \multicolumn{2}{|l|}{$\mathrm{PNC}$} & \multirow{2}{*}{$\begin{array}{l}\text { Women's } \\
\text { empowerment } \\
\text { and healthcare }\end{array}$} \\
\hline & $\mathrm{AOR}(95 \% \mathrm{CI})$ & $\begin{array}{c}p- \\
\text { value }\end{array}$ & AOR $(95 \%$ CI) & $\begin{array}{c}p- \\
\text { value }\end{array}$ & $\mathrm{AOR}(95 \% \mathrm{CI})$ & $\begin{array}{c}p- \\
\text { value }\end{array}$ & \\
\hline \multicolumn{7}{|l|}{ Year } & \\
\hline \multirow{2}{*}{2014} & 1.31 (1.19-1.44) & 0.000 & $1.85(1.68-2.03)$ & 0.000 & $2.47(2.27-2.70)$ & 0.000 & \\
\hline & 1.00 & & 1.00 & & 1.00 & & \\
\hline \multirow{3}{*}{$\begin{array}{l}\text { Age (years) } \\
21-30 \\
>30 \\
\leq 20^{\mathrm{R}}\end{array}$} & & & & & & & 1113 \\
\hline & $\begin{array}{l}1.07(0.94-1.12) \\
1.23(1.01-1.49)\end{array}$ & $\begin{array}{l}0.286 \\
0.036\end{array}$ & $\begin{array}{l}1.32(1.16-1.50) \\
1.82(1.49-2.21)\end{array}$ & $\begin{array}{l}0.000 \\
0.000\end{array}$ & $\begin{array}{l}1.07(0.95-1.21) \\
1.27(1.06-1.51)\end{array}$ & $\begin{array}{l}0.249 \\
0.008\end{array}$ & \\
\hline & 1.00 & & 1.00 & & 1.00 & & \\
\hline \multicolumn{8}{|l|}{ Wealth index } \\
\hline Poorer & $1.09(0.92-1.30)$ & 0.299 & $1.27(1.07-1.51)$ & 0.006 & $1.15(1.01-1.31)$ & 0.029 & \\
\hline Middle & $1.31(1.10-1.56)$ & 0.002 & $1.69(1.42-2.01)$ & 0.000 & $1.40(1.22-1.61)$ & 0.000 & \\
\hline Richer & $1.77(1.48-2.11)$ & 0.000 & $2.43(2.04-2.89)$ & 0.000 & $2.08(1.79-2.40)$ & 0.000 & \\
\hline Richest & $3.46(2.84-4.20)$ & 0.000 & $4.61(3.79-5.62)$ & 0.000 & $3.80(3.17-4.56)$ & 0.000 & \\
\hline Poorest $^{R}$ & 1.00 & & 1.00 & & 1.00 & & \\
\hline \multicolumn{8}{|c|}{ Husband's education } \\
\hline Primary & $1.05(0.90-1.21)$ & 0.488 & $1.14(0.98-1.31)$ & 0.069 & $1.04(0.92-1.16)$ & 0.488 & \\
\hline Secondary & $1.24(1.07-1.44)$ & 0.004 & $1.40(1.21-1.63)$ & 0.000 & $1.29(1.13-1.46)$ & 0.000 & \\
\hline Higher & $1.89(1.58-2.27)$ & 0.000 & $2.60(2.18-3.11)$ & 0.000 & $2.23(1.87-2.65)$ & 0.000 & \\
\hline No education $^{\mathrm{R}}$ & & & 1.00 & & 1.00 & & \\
\hline \multicolumn{8}{|c|}{ Wanted index child } \\
\hline Yes & $1.13(1.01-1.26)$ & 0.031 & $1.05(0.93-1.17)$ & 0.410 & $1.03(0.93-1.13)$ & 0.501 & \\
\hline & 1.00 & & 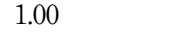 & & 1.00 & & \\
\hline \multicolumn{8}{|l|}{ Parity } \\
\hline 2nd & $0.81(0.72-0.92)$ & 0.001 & $0.58(0.52-0.66)$ & 0.000 & $0.71(0.629-0.75)$ & 0.000 & \\
\hline & $0.68(0.58-0.80)$ & 0.000 & $0.49(0.42-0.58)$ & 0.000 & $0.67(0.58-0.78)$ & 0.000 & \\
\hline $3+$ & $0.47(0.38-0.59)$ & 0.000 & $0.31(0.25-0.38)$ & 0.000 & $0.61(0.51-0.72)$ & 0.000 & \\
\hline & 1.00 & & 1.00 & & & & \\
\hline \multicolumn{8}{|l|}{ Sex of child } \\
\hline \multicolumn{3}{|l|}{$\begin{array}{l}\text { Female } \\
\text { Male }^{R}\end{array}$} & $\begin{array}{l}0.91(0.83-1.00) \\
1.00\end{array}$ & 0.057 & & & \\
\hline \multicolumn{8}{|l|}{$K L I$} \\
\hline Medium & $1.56(1.34-1.81)$ & 0.000 & $1.19(1.03-1.38)$ & 0.014 & $1.16(1.03-1.31)$ & 0.009 & \\
\hline High & $2.14(1.83-2.51)$ & 0.000 & $1.71(1.47-1.99)$ & 0.000 & $1.56(1.37-1.77)$ & 0.000 & \\
\hline Low $^{R}$ & 1.00 & & 1.00 & & 1.00 & & \\
\hline \multicolumn{8}{|l|}{$A T P V I$} \\
\hline \multicolumn{8}{|c|}{$1.05(0.83-1.31)$} \\
\hline \multirow{2}{*}{\multicolumn{8}{|c|}{1.00}} \\
\hline & & & & & & & \\
\hline \multicolumn{8}{|l|}{$D M P I$} \\
\hline Medium & $1.19(1.02-1.38)$ & 0.022 & $1.14(0.98-1.32)$ & 0.080 & $1.18(1.03-1.35)$ & 0.017 & \\
\hline High & $1.25(1.13-1.39)$ & 0.000 & $1.12(1.01-1.24)$ & 0.029 & $1.16(1.05-1.27)$ & 0.002 & \\
\hline Low $^{R}$ & 1.00 & & 1.00 & & 1.00 & & \\
\hline \multirow{5}{*}{$\begin{array}{l}\text { LFPI } \\
\text { Medium } \\
\text { High } \\
\text { Low }^{R}\end{array}$} & & & & & & & $\begin{array}{r}\text { Table } 3 . \\
\text { Three-level logistic }\end{array}$ \\
\hline & & & $0.89(0.60-1.33)$ & 0.583 & $1.36(0.96-1.92)$ & 0.074 & Inree-level $10 \mathrm{gistic}$ \\
\hline & & & $0.76(0.67-0.88)$ & 0.000 & $1.25(1.11-1.92)$ & 0.000 & finding potential \\
\hline & & & 1.00 & & 1.00 & & factors associated with \\
\hline & & & & & \multicolumn{2}{|c|}{ (continued) } & and PNC \\
\hline
\end{tabular}




\begin{tabular}{|c|c|c|c|c|c|c|c|}
\hline \multirow{2}{*}{$\begin{array}{l}\text { JHR } \\
36,6\end{array}$} & \multirow[b]{2}{*}{ Covariates } & \multicolumn{2}{|l|}{ ANC } & \multicolumn{2}{|c|}{ Facility delivery } & \multicolumn{2}{|l|}{ PNC } \\
\hline & & AOR $(95 \% \mathrm{CI})$ & $\begin{array}{c}p- \\
\text { value }\end{array}$ & $\mathrm{AOR}(95 \% \mathrm{CI})$ & $\begin{array}{c}p- \\
\text { value }\end{array}$ & $\mathrm{AOR}(95 \% \mathrm{CI})$ & $\begin{array}{c}p- \\
\text { value }\end{array}$ \\
\hline & Constant & $0.08(0.05-0.11)$ & 0.000 & $0.10(0.06-0.14)$ & 0.000 & $0.29(0.21-0.41)$ & 0.000 \\
\hline \multirow[t]{2}{*}{1114} & $\begin{array}{l}\text { Random effects } \\
\text { Division } \\
\text { Place of resid }\end{array}$ & $\begin{array}{l}0.23(0.056-0.923) \\
0.35(0.202-0.652)\end{array}$ & & $\begin{array}{l}0.16(0.01-2.34) \\
0.38(0.21-0.66)\end{array}$ & & $\begin{array}{l}0.11(0.009-1.30) \\
0.22(0.11-0.42)\end{array}$ & \\
\hline & $\begin{array}{l}\text { ICC } \\
\text { Division } \\
\text { Place of resident } \\
\text { |division }\end{array}$ & $\begin{array}{l}0.014 \\
0.051\end{array}$ & & $\begin{array}{l}0.007 \\
0.05\end{array}$ & & $\begin{array}{l}0.004 \\
0.02\end{array}$ & \\
\hline & Note(s): ${ }^{R}$ Refere & ttegory, $\mathrm{AOR}=$ adj & sted od & ratio, $\mathrm{CI}=$ confi & ence in & & \\
\hline
\end{tabular}

deliveries with unskilled obstetricians often leads to complications and results in mothers needing emergency medical support. However, the prevalence of the practice of at least four ANC visits and facility delivery was still below $50 \%$, and the practice of PNC visits was developing towards universal practice. Although this study found an increase in the percentage of highly empowered women in KLI, ATPVI and LFPI, the percentage of highly empowered women decreased in the case of DMPI. This decrease is a matter of concern and requires urgent action.

Women with higher knowledge levels were found to be significantly associated with the utilization of at least four ANC visits, facility delivery and PNC visit. This result is obvious as mothers with higher knowledge know more about maternal and child health and are, therefore, more conscious about the utilization of maternal healthcare facilities. A similar result was reported in previous studies [15-17].

Women who possess high autonomy regarding decision-making are expected to have more access to healthcare facilities. Previous studies also suggested women's decisionmaking autonomy increased the utilization of maternal healthcare facilities [18, 19]. A study in India found women's access to family resources (ability to save money) influenced the use of maternal healthcare facilities [20]. Women who had high levels of empowerment in the DMPI were also found to have higher levels of access to utilization of at least four ANC visits, facility delivery and PNC visits within two days of delivery.

Consistent with a previous study, our study also revealed that women who strongly disagreed with wife-beating justification (i.e. high value for ATPVI) were more likely to have at least four ANC visits and facility delivery [21]. This study also found a higher prevalence of PNC visits for mothers with high ATPVI values. Mothers who disagreed with wife-beating justification had a sense of self-esteem, had a greater sense of entitlement and had more access to healthcare facilities.

Surprisingly, this study found women with high empowerment in labor force participation were less likely to have a facility delivery. Other studies also found unemployed mothers had a higher chance of receiving professional delivery care services compared to the employed mothers $[21,22]$. We tried to find a plausible explanation for this finding. The level of education of mothers may be the reason for this finding as we found employed mothers had a lower level of education compared to the unemployed mothers (result not reported). In the case of PNC visits, we found that highly empowered mothers in the LFPI were more prone to have PNC visits compared to the low empowered mothers. The explanation of this finding lies in the prevalence of facility delivery. Mothers with high values of LFPI gave birth without a delivery facility and faced more complications immediately after the delivery, making it necessary to visit a doctor or physician. 
The legalized age of marriage for Bangladeshi girls is at least 18 years old. Several studies stated the negative consequences of early marriage [23-25]. For instance, early married women have less autonomy in decision-making and face higher levels of partner violence and are, therefore, expected to have lower access to healthcare facilities. On the other hand, older women become more prominent as a mother and achieve a status in the family and community that may increase the utilization of healthcare facilities [26, 27]. Consistent with previous studies, our study also revealed that older mothers were more likely to utilize healthcare facilities than younger mothers $[15,21]$.

Similar to previous studies, this study also found women from wealthier families and whose partners were more educated had a higher chance of utilizing healthcare facilities [15, $21,28,29]$. High educated husbands are more aware of their wives' health and child health and influence their wives to get healthcare facilities. Also, wealthy families have an additional amount of money to spend on healthcare, which may explain why mothers from wealthier backgrounds may have a higher chance of access to healthcare facilities. Additionally, having more children creates resource constraints in the family with the result that the mothers' accessibility to healthcare facilities reduced [30]. This study also found a similar result that mothers with more parity had a lower rate of utilization of healthcare facilities.

\section{Limitation and strength of study}

Since the data sets were obtained from cross-sectional studies, we were unable to demonstrate a cause-effect relationship. Being a retrospective study, there was a chance of recall bias. We were not able to use the more recent BDHS 2017 data set as this data set was not publicly available at the time of our research. Using this data set could give us a more recent scenario of the considering issues. The strength of this study is that it used BDHS 2011 and 2014 data sets that are nationally representative.

\section{Conclusion}

Although the practice of healthcare facilities was found to be increasing, they remain far behind the universal practice. Women's empowerment was found to significantly increase the utilization of healthcare facilities along with other socio-economic and demographic factors. Special attention must be given to women's decision-making autonomy as it was found to decrease from 2011 to 2014. This study would be of relevance to governmental and NGOs in the effort to develop more effective intervention programs to empower women to utilize maternal healthcare facilities.

Conflict of Interest: None

\section{References}

1. Asamoah BO, Moussa KM, Stafström M, Musinguzi G. Distribution of causes of maternal mortality among different socio-demographic groups in Ghana; a descriptive study. BMC Public Health. 2011; 11: 159. doi: 10.1186/1471-2458-11-159.

2. El Arifeen S, Hill K, Ahsan KZ, Jamil K, Nahar Q, Streatfield PK. Maternal mortality in Bangladesh: a countdown to 2015 country case study. Lancet. 2014; 384(9951): 1366-74. doi: 10. 1016/s0140-6736(14)60955-7.

3. Shahabuddin AS, Delvaux T, Abouchadi S, Sarker M, De Brouwere V. Utilization of maternal health services among adolescent women in Bangladesh: a scoping review of the literature. Trop Med Int Health. 2015; 20(7): 822-9. doi: 10.1111/tmi.12503.

4. Bangladesh, Ministry of Health and Family Welfare. Partnership for maternal, newborn \& child health; world health organization [WHO]; world bank; alliance for health policy and systems research. Success factors for women's and children's health: Bangladesh. Geneva: WHO; 2015. 
JHR

36,6

1116

5. World Health Organization [WHO]. WHO recommendations on antenatal care for a positive pregnancy experience. Geneva: WHO; 2016.

6. National Institute of Population Research and Training [NIPORT]; ICF. Bangladesh demographic and health survey 2017. Dhaka, Bangladesh and Rockville, Maryland: NIPORT, and ICF; 2019.

7. Phan L. Measuring women's empowerment at household level using DHS data of four Southeast Asian countries. Soc Indic Res. 2016; 126(1): 359-78. doi: 10.1007/s11205-015-0876-y.

8. Sebayang SK, Efendi F, Astutik E. Women's empowerment and the use of antenatal care services: analysis of demographic health surveys in five Southeast Asian countries. Women Health. 2019; 59(10): 1155-71. doi: 10.1080/03630242.2019.1593282.

9. Rahman A, Nisha MK, Begum T, Ahmed S, Alam N, Anwar I. Trends, determinants and inequities of $4^{+}$ANC utilisation in Bangladesh. J Health Popul Nutr. 2017; 36(1): 2. doi: 10.1186/ s41043-016-0078-5.

10. Chanda SK, Ahammed B, Howlader MH, Ashikuzzaman M, Shovo TE, Hossain MT. Factors associating different antenatal care contacts of women: a cross-sectional analysis of Bangladesh demographic and health survey 2014 data. PloS One. 2020; 15(4): e0232257. doi: 10.1371/journal. pone.0232257.

11. National Institute of Population Research and Training [NIPORT]; Mitra and Associates; ICF International. Bangladesh demographic and health survey 2011. Dhaka, Bangladesh and Calverton, Maryland: NIPORT, Mitra and Associates, and ICF International; 2013.

12. National Institute of Population Research and Training [NIPORT]; Mitra and Associates; ICF International. Bangladesh demographic and health survey 2014. Dhaka, Bangladesh and Rockville, Maryland: NIPORT, Mitra and Associates, and ICF International; 2016.

13. Agresti A. Alternative modeling of binary response data. In: Categorical data analysis. 3rd ed. Hoboken, New Jerssey, NJ: John Wiley \& Sons; 2013. 251-92.

14. Rabe-Hesketh S, Skrondal A. Multilevel and longitudinal modeling using Stata, Volume II: categorical responses, counts, and survival. 3rd ed. College Station, TX: Stata Press; 2012.

15. Haque SE, Rahman M, Mostofa MG, Zahan MS. Reproductive health care utilization among young mothers in Bangladesh: Does autonomy matter? Wom. Health Issues. 2012; 22(2): e171-80. doi: 10. 1016/j.whi.2011.08.004.

16. Amin R, Shah NM, Becker S. Socioeconomic factors differentiating maternal and child healthseeking behavior in rural Bangladesh: a cross-sectional analysis. Int. J. Equity Health. 2010; 9: 9. doi: 10.1186/1475-9276-9-9.

17. Chakraborty N, Islam MA, Chowdhury RI, Bari W, Akhter HH. Determinants of the use of maternal health services in rural Bangladesh. Health Promotion International. 2003; 18(4): 327-37. doi: 10.1093/heapro/dag414.

18. Adhikari R. Effect of women's autonomy on maternal health service utilization in Nepal: a cross sectional study. BMC Wom. Health. 2016; 16: 26. doi: 10.1186/s12905-016-0305-7.

19. Sado L, Spaho A, Hotchkiss DR. The influence of women's empowerment on maternal health care utilization: evidence from Albania. Soc Sci Med. 2014; 114: 169-77. doi: 10.1016/j.socscimed.2014.05.047.

20. Maitra P. Parental bargaining, health inputs and child mortality in India. J Health Econ. 2004; 23(2): 259-91. doi: 10.1016/j.jhealeco.2003.09.002.

21. Tareque MI, Alam MS, Peet ED, Rahman MM, Rahman KMM. Justification of wife beating and utilization of antenatal and delivery care in Bangladesh. J. Interpers Violence. February 2020. doi: $10.1177 / 0886260519898444$.

22. Ghose B, Feng D, Tang S, Yaya S, He Z, Udenigwe O, et al. Women's decision-making autonomy and utilisation of maternal healthcare services: results from the Bangladesh demographic and health survey. BMJ Open. 2017; 7(9): e017142. doi: 10.1136/bmjopen-2017-017142.

23. Islam TM, Tareque MI, Tiedt AD, Hoque N. The intergenerational transmission of intimate partner violence in Bangladesh. Glob. Health Action. 2014; 7(1): 23591. doi: 10.3402/gha.v7.23591. 
24. Islam TM, Tareque MI, Sugawa M, Kawahara K. Correlates of intimate partner violence against women in Bangladesh. J Fam Violence. 2015; 30(4): 433-44. doi: 10.1007/s10896-015-9683-0.

25. Jensen R, Thornton R. Early female marriage in the developing world. Gend Dev. 2003; 11(2): 9-19. doi: 10.1080/741954311.

26. Kishor S, Johnson K. Profiling domestic violence: a multi-country study. Calverton, MD: ORC Macro; 2004.

27. Mainuddin A, Ara Begum H, Rawal LB, Islam A, Shariful Islam SM. Women empowerment and its relation with health seeking behavior in Bangladesh. J Family Reprod Health. 2015; 9(2): 65-73.

Women's empowerment and healthcare facilities

28. Dalal K, Shabnam J, Andrews-Chavez J, Martensson LB, Timpka T. Economic empowerment of women and utilization of maternal delivery care in Bangladesh. Int J Prev Med. 2012; 3(9): 628-36.

29. Anwar I, Nababan HY, Mostari S, Rahman A, Khan JA. Trends and inequities in use of maternal health care services in Bangladesh, 1991-2011. PloS One. 2015; 10(3): e0120309. doi: 10.1371/ journal.pone.0120309.

30. Pebley AR, Goldman N, Rodríguez G. Prenatal and delivery care and childhood immunization in Guatemala: Do family and community matter?. Demography. 1996; 33(2): 231-47.

\section{Corresponding author}

Foyez Ahmmed can be contacted at: foyez.sbi@gmail.com

For instructions on how to order reprints of this article, please visit our website: 